\title{
Adenite sebácea em cães: relato de três casos
}

\author{
[Sebaceous adenitis in dogs: report of three cases]
}

\section{"Relato de Caso/Case Report"}

\author{
Priscila Canato Schellin ${ }^{1}$, Rafaela Rocha Amorim Barros², Paula Felex Montenegro², \\ Maria Helena Barros Tavares ${ }^{1}$, Edna Michelly Sá Santos ${ }^{1, *}$
}

\author{
${ }^{1}$ Departamento de Medicina Veterinária, Universidade Federal Rural de Pernambuco, Recife-PE, Brasil. \\ ${ }^{2}$ Médica veterinária autônoma, Recife-PE, Brasil., Recife-PE, Brasil. \\ *Autor para correspondência/Corresponding author: E-mail: ednamichelly@ hotmail.com
}

\begin{abstract}
Resumo
A adenite sebácea é uma dermatose incomum em cães, caracterizada por ser uma doença inflamatória que resulta na destruição da glândula sebácea. Embora possa ocorrer em muitas raças, existe uma aparente prevalência em Poodles standard, Vizslas, Akitas e Samoiedas, variando a severidade dos sintomas de acordo com a raça e o tipo de pelagem. O diagnóstico se baseia nos sinais clínicos apresentados pelo paciente e nos achados do exame histopatológico das lesões, que demonstram ausência de glândulas sebáceas e presença de inflamação mínima. A doença é incurável, porém o tratamento pode ser realizado pela aplicação de óleos nas áreas acometidas e administração de imunomoduladores sistêmicos de maneira ininterrupta, evitando assim a recidiva dos sinais clínicos. Objetivou-se descrever três casos de adenite sebácea em cães nos quais os animais apresentaram lesões alopécicas, com formação melicérica multifocais e prurido com intensidade variável de ausente a moderada. $\mathrm{O}$ diagnóstico definitivo foi obtido pelo exame histopatológico e o tratamento instituído consistiu da aplicação tópica de ciclosporina diluída em óleo mineral, ou óleo mineral isolado. Pode-se concluir que foi possível diagnosticar a adenite sebácea nos casos descritos, por meio de dados da anamnese, exame clínico e exames complementares. Além disso, vale salientar que os cães malteses não estão listados como predispostos a enfermidade e a utilização da terapia tópica com óleo mineral associado ou não com ciclosporina demonstrou-se ser eficaz no tratamento da adenite sebácea de cães na sua fase crônica.
\end{abstract}

Palavras-Chave: glândula sebácea, óleo, Maltês.

\begin{abstract}
Sebaceous adenitis is an unusual dermatosis in dogs, characterized by being an inflammatory disease that results in the destruction of the sebaceous gland. Although it occurs in many breeds, there is an apparent prevalence in standard Poodles, Vizslas, Akitas, and Samoiedas, and the severity of symptoms varies according to breed and coat type. The diagnosis is based on clinical signs presented by the patient and also depend on the histopathological examination of the lesions, which shows absence of sebaceous glands and presence of minimal inflammation. The disease is incurable, but its treatment can be performed by applying oils in the affected areas and also by administering systemic immunomodulators continually to avoid relapse. The present work aims to describe three cases of sebaceous adenitis in dogs, in which the animals presented alopecic lesions, with multifocal meliceric formation and moderate to intense pruritus. The definitive diagnosis was obtained through histopathological examination and the treatment consisted of topical application of mineral oil, or application of cyclosporin diluted in mineral oil. The diagnostic of sebaceous adenitis in the cases described was possible due anamnesis data, clinical examination and complementary tests. In addition, it is worth noting that Maltese dogs are not listed as predisposed to the disease and the use of topical therapy with mineral oil associated with or without cyclosporine has been shown to be effective in the treatment of sebaceous adenitis in dogs in their chronic phase.
\end{abstract}

Key-words: sebaceous gland, oil, Maltese. 


\section{Introdução}

Adenite sebácea é um distúrbio disqueratótico crônico, caracterizado pela degeneração e atrofia pós-inflamatória das glândulas sebáceas (Vercelli et al., 2004; Lowell, 2005; Mendleu e Hnilica, 2006; Grognet, 2008). Trata-se de uma dermatopatia incomum, cuja etiologia e patogenia ainda não estão bem definidas (Linek, 2008; Medleau e Hnilica, 2009).

Não há predileção por sexo, podendo atingir cães adultos jovens e de meia idade. Várias raças têm sido descritas como predispostas a doença (Larsson e Lucas, 2016), principalmente Poodle Standard, Akita, Samoieda, Viszla (Dustan e Hargis, 1992; Farias et al., 2000; Scott et al., 2001; Mendleu e Keith, 2003; Mendleu e Hnilica, 2006; Obladen et al., 2007) e Lhasa Apso (Obladen et al., 2007; Miller et al., 2013). Segundo Frazer et al. (2011), os cães havaneses também são predispostos a esta dermatopatia.

As hipóteses relacionadas à patogenia da adenite sebácea incluem alterações genéticas estruturais primárias da glândula ou dos ductos sebáceos (Farias et al., 2000; Reichler et al., 2001; Scott et al., 2001), resposta imunomediada contra antígenos presentes nos componentes da glândula ou da secreção sebácea (Scott et al., 1996); anormalidades da cornificação, ou defeito no metabolismo dos lipídeos (Scott et al., 1996; Reichler et al., 2001). A adenite sebácea também pode se desenvolver secundariamente a determinadas afecções, sendo observada em várias doenças de base, tais como leishmaniose, demodiciose, alergia alimentar e síndromes úveodermatológicas (Gross et al., 1992; White et al., 1995).

Os sinais clínicos são variáveis, e dependem do tipo de pelagem e raça do animal (Scott et al., 2001). Segundo Farias et al. (2000), as manifestações clínicas mais comuns da adenite sebáceas são descamação progressiva simétrica e alopecia associada à seborreia seca generalizada. As áreas mais comumente acometidas são as orelhas, face, cabeça e tronco (Scott et al. 2001; Mendleu e Keith, 2003). O prurido normalmente não está presente, mas nos casos em que existe infecção secundária por bactérias ou malassezia, pode ser observado (Sousa, 2006; Tevell et al., 2008).

Os diagnósticos diferenciais para lesões hiperqueratóticas oriundas da adenite sebácea devem incluir seborreia primária, dermatose responsiva à vitamina A foliculite estafilocócica, demodicidose e dermatofitose (Scott et al.,1996).

O diagnóstico da adenite sebácea se baseia na raça do animal, nos sinais clínicos e nos achados da análise histopatológica de fragmentos da pele (Obladen et al., 2007), onde a ausência de glândulas sebáceas é o principal achado que diferencia a adenite sebácea das outras enfermidades (Gross et al., 2009). O tricograma pode ser utilizado como exame de triagem para os pacientes com suspeita da enfermidade, uma vez que a formação de cilindros foliculares pode estar presente (Scott et al., 2001).

Os tratamentos utilizados podem ser tópicos e sistêmicos, podendo ser usados isolados ou em combinação. Quando existe piodermite secundária, o seu tratamento também se faz necessário (White et al., 2000). Dentre os produtos tópicos, citam-se xampus queratolíticos a base de enxofre e ácido tjhu1salicílico, enxagues emolientes (Scott et al., 2001; Mendleu et al., 2003; Vercelli et al., 2004; Lowell, 2005), propilenoglicol em concentrações de 50 a 75\% (Rhodes e Corner, 2005; Lucas et al., 2008), além de óleos hidratantes para bebês seguida da utilização de xampus múltiplos para remoção do óleo e escamas (Lowell, 2005; Rhodes e Codner, 2005). Além disso, a utilização de imunomoduladores como a ciclosporina dissolvida em óleo mineral e utilizada topicamente foi descrita com bons resultados terapêuticos (Lucas et al., 2008). Como tratamento sistêmico, citam-se produtos contendo ácidos graxos ômega-3 e ômega-6 (Griffin et al., 1993; Scott, 1993), retinoides sintéticos (White, 1995; Miller et al., 2013) e utilização adjunta de vitamina A (Sousa, 2006).

É uma dermatopatia incurável cujo prognóstico é variável, dependendo da gravidade da doença, porém o diagnóstico e tratamento precoce melhoram o prognóstico (Mendleu e Keith, 2003). Tendo em vista que a adenite sebácea é uma dermatopatia incomum, objetiva-se com esse trabalho relatar três casos dessa afecção em cães, sendo dois da raça Maltês e um sem raça definida (SRD).

\section{Descrição dos Casos}

Previamente, é importante estabelecer que em todos os casos como exames complementares, foram realizados exame parasitológico de raspado cutâneo e exame tricográfico das lesões. Não foram observados ácaros, bem como esporos fúngicos no exame parasitológico, porém no tricográfico, foi 
possível observar a presença de cilindros foliculares. A partir desses achados, a suspeita clínica foi de adenite sebácea. Para diagnóstico definitivo, foi realizado o exame histopatológico, que demonstrou ausência de glândulas sebáceas nas amostras cutâneas, caracterizando a doença em sua fase crônica.

\section{Caso 1}

Uma cadela da raça Maltês, castrada e com cinco anos de idade, foi atendida em um consultório particular na cidade de Recife, Pernambuco. Durante a anamnese, a tutora relatou alterações dermatológicas, alopecia e prurido moderado há seis meses. A paciente já havia sido tratada com griseofulvina e cefalexina por mais de 20 dias, sem melhora clínica. A alimentação consistia de ração comercial Super Premium e frango cozido. Dosagens hormonais de T4 livre, T4 total, hormônio estimulador da tireoide e o teste de supressão por dexametasona, solicitadas por médico veterinário anterior, estavam dentro dos parâmetros de normalidade.

Ao exame físico, pôde-se observar eritema e descamação de bordas e face dorsal das orelhas que evoluíam para a região cervical dorsal com escamas bem aderidas, porém condutos normais. Foi possível observar extensa alopecia dessas regiões com presença de pelos curtos e frágeis. Além disso, eram evidentes lesões semelhantes multifocais dorsais (Figura 1).

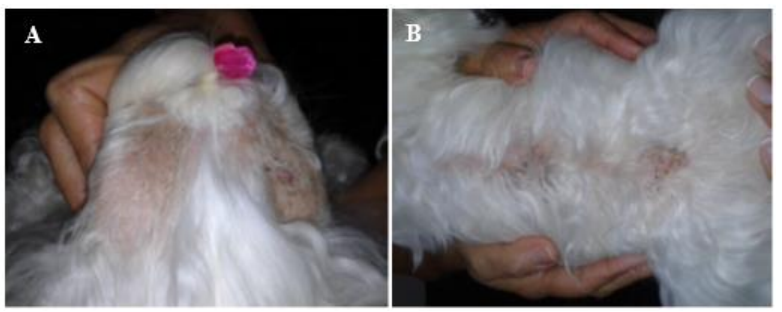

Figura 1. Eritema, descamação e alopecia em A) face dorsal das orelhas e B) região dorsal da cadela da raça Maltês.

Foi instituído o tratamento com o uso de ciclosporina diluída em óleo mineral (Lucas et al., 2008) para ser aplicada nas áreas das lesões a cada quatro dias, deixando agir por 1 hora, seguido por banho com xampu desengordurante a base de alcatrão 4\%. Após três meses de tratamento, houve melhora acentuada, o paciente repilou todas as lesões primárias (Figura 2), porém ainda foi possível observar leve presença de material sebáceo sobre a pele nessas regiões e pelos ligeiramente amarelados. Instruiu-se a continuação do tratamento, desta vez, uma vez por semana como terapia de manutenção.

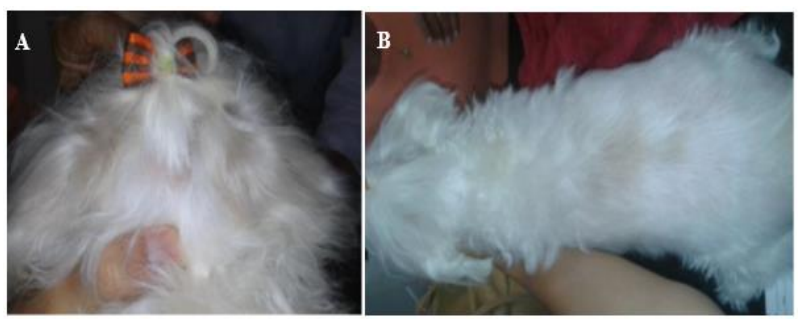

Figura 2. Repilação das lesões primárias em A) face dorsal das orelhas e B) região dorsal de cadela da raça Maltês após tratamento para adenite sebácea.

\section{Caso 2}

Um canino, macho, da raça Maltês de cinco anos, foi atendido em consultório particular da cidade de Recife, Pernambuco, com histórico de perda de pelo progressiva há mais de três meses e prurido leve. Durante a anamnese, a tutora informou que o paciente apresentava discreto prurido e que já tinha feito uso de cefalexina, ômega 3 e imunoestimulantes, sem melhora clínica. A alimentação consistia em ração Premium, adicionada a frango, carne bovina ou peixe. Os banhos eram realizados semanalmente com xampu à base de peróxido de benzoíla 2,5\% .

Ao exame físico, pôde-se observar extensa alopecia em toda a região cervical e dorsal (Figura 3), com material sebáceo aderido à pele, formado de pequenas crostas melicéricas, inclusive na ponta das orelhas. O paciente também apresentava algumas áreas de foliculite dorsal.

Foi instituído o tratamento com o uso de óleo mineral para ser aplicado nas áreas com lesões a cada quatro dias, deixando agir por 2 horas, seguido por banho com xampu desengordurante a base de alcatrão $4 \%$. O animal retornou após um mês de tratamento, apresentando a pele mais hidratada e fina, com alguns pelos em crescimento. Após três meses da reavaliação, a tutora relatou melhora acentuada e repilação total das lesões, instruindo-se a continuação do tratamento.

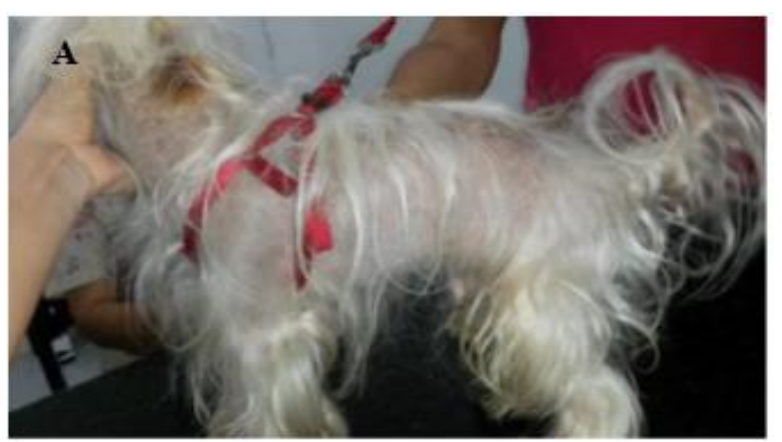

Figura 3. Extensa alopecia em região cervical e dorsal de cão da raça Maltês. 


\section{Caso 3}

Um cão macho, SRD, com cinco anos de idade, resgatado na rua há dois anos, foi atendido no Hospital Veterinário da Universidade Federal de Pernambuco (UFRPE), com histórico de rarefação pilosa bilateral dos flancos e ausência de prurido há nove meses. Foi relatado que este se alimentava de ração e esporadicamente baço bovino, e que o mesmo convivia com outros nove cães que não apresentavam lesão de pele. $O$ animal foi submetido a um tratamento prévio com itraconazol durante um mês, sem melhora dos sinais clínicos.

Ao exame físico do paciente, foi observada hipotricose bilateral simétrica em região lateral à coluna espinhal (Figura 4A) se estendendo para região de gradil costal e flanco, membros e bordas das orelhas. Além disso, foi possível observar um conteúdo sero-sebáceo aderido aos pelos. Ainda nas áreas de hipotricose dorsal, pôde-se observar a presença de pequenos pelos com crescimento retardado e aderidos à pele entremeados a uma formação melicérica.

$\mathrm{O}$ protocolo terapêutico instituído foi a aplicação semanal de óleo mineral nas áreas afetadas, deixando o produto em contato com a pele durante 2 horas. Em seguida, banhos semanais com produtos anti-seborreicos compostos por ácido salicílico a $2 \%$, enxofre a $2 \%$ e alcatrão a $4 \%$. Após 40 dias do início do tratamento, o paciente apresentou melhora significativa e o plano terapêutico foi mantido. $\mathrm{Na}$ ocasião, notou-se início de repilação na região dorsal (Fig. 4B) e membros, bem como o desaparecimento do material sero-sebáceo aderido aos pelos e dos cilindros foliculares.

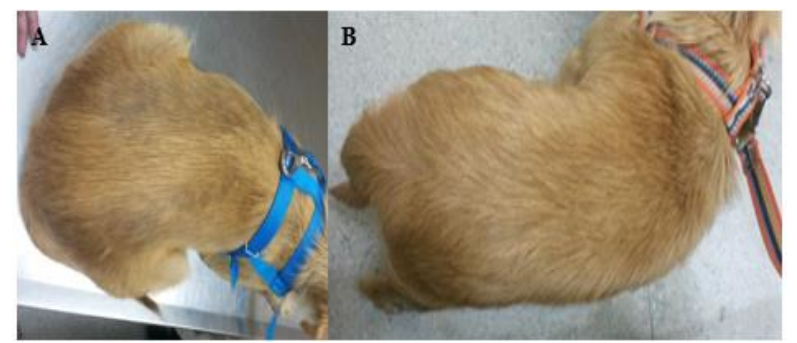

Figura 4. A) Hipotricose bilateral simétrica em região lateral à coluna espinhal, B) Início de repilação bilateral simétrica em região lateral à coluna espinhal no cão sem raça definida (SRD).

\section{Discussão}

A adenite sebácea é uma doença inflamatória idiopática das glândulas sebáceas que, segundo Griffin et al. (1993), tende a acometer os cães adultos jovens e de meia-idade, o que corrobora com os casos descritos acima, onde todos os pacientes possuíam cinco anos de idade. Apesar de ser relatada uma maior incidência nos cães das raças Vizslas, Akitas, Samoyedoas e Poodles (Griffin et al., 1993), dos três cães apresentados, dois eram da raça Maltês e um era SRD. Este último, por sua vez, condiz com o relatado por Reis (2009), que diagnosticou adenite sebácea em um cão mestiço, em Curitiba, Paraná.

Os sinais clínicos de alopecia e formação de material sebáceo localizados em região cervical, dorsal e caudal apresentados nos cães da raça Maltês são compatíveis aos descritos por Reis (2009). O cão SRD apresentou lesões na região de tronco, membros e orelhas, discordando do que descreveram Scott et al. (2001), cujas áreas acometidas também envolviam a face e cabeça. Neste relato, o cão sem raça definida não apresentava prurido da mesma forma como foi descrito por Travell et al. (2008), todavia, os cães da raça Maltês desenvolveram prurido de intensidades leve e moderada, contradizendo os autores supracitados.

Foi sugerido que o tipo de pelagem poderia interferir na manifestação clínica da doença, sendo que os cães de pelos longos apresentam principalmente hiperqueratose difusa, seborreia e plugs foliculares (Sousa, 2006; Miller et al., 2013), o que corrobora com o observado nos cães da raça Maltês. Por outro lado, tais sinais também foram observados no caso do cão SRD, que, por sua vez, apresentava pelo curto. Segundo Obladen et al. (2007), a mesma apresentação clínica foi citada nos cães da raça Lhasa Apso.

A presença de cilindros foliculares no exame tricográfico descrito por Larsson e Lucas (2016), além dos sinais clínicos e histórico dos pacientes, sugeriram o diagnóstico presuntivo da enfermidade, entretanto o diagnóstico definitivo foi estabelecido de acordo com o proposto por Linek (2008), baseando-se na exclusão dos diagnósticos diferenciais e no exame histopatológico das lesões.

No resultado da análise da biopsia da pele, o diagnóstico de adenite sebácea foi confirmado. Nos fragmentos analisados, observou-se atrofia de bulbo piloso com formações císticas em alguns folículos, glândulas sudoríparas preservadas e ausência de glândulas sebáceas e de infiltrado inflamatório. Tais achados corroboram o descrito por Gross et al. (2005), que além de relatarem ausência das glândulas sebáceas em casos crônicos, e possibilidade de presença ou ausência de reação inflamatória, também descreveram que esta é incomumente substituída por fibrose perifolicular. 
Devido à cronicidade dos casos relatados, a terapia sistêmica não foi instituída, baseada no que foi descrito por Lam et al. (2011), que relatou a melhor eficácia de fármacos sistêmicos na fase aguda da doença. Assim como no caso do autor supracitado, foi realizada a terapia tópica, que apresentou efeitos satisfatórios nos três relatos. A constatação da eficácia nos três casos descritos corrobora com os resultados apresentados por Lowell (2005) e Lucas et al. (2008).

\section{Conclusão}

Foi possível diagnosticar a adenite sebácea nos três casos descritos de adenite sebácea, por meio de dados da anamnese, exame clínico e exames complementares.

Além disso, os cães da raça Maltês podem ser acometidos pela adenite sebácea e a utilização da terapia tópica a base de óleo mineral com ou sem ciclosporina demonstra ser eficaz no tratamento dos três cães diagnosticados com a enfermidade em sua fase crônica.

\section{Conflito de interesse}

Os autores declaram não existir conflito de interesse.

\section{Referências}

Farias, M.R.; Peres, J.A.; Fabris, V.E.; Costa, F.S.; Pinto, R.G. Adenite sebácea granulomatosa em cães da raça akita. Revista Clínica Veterinária, 25: 33-38, 2000.

Frazer, M.; Schick, A.; Lewis, T.; Jazic, E. Sebaceous adenitis in Havanese dogs: a retrospective study of the clinical presentation and incidence. Veterinary Dermatology, 22(3): 267-274, 2010.

Griffin, C.E.K.; MacDonald, K.W.; John, M.; Griffin, C.E.; Kwochka, K.W.; MacDonald, J. M. Current veterinary dermatology: the science and art of therapy. Michigan: Mosby Year Book, 1993. 378p.

Grognet, J. Sebaceous adenitis. Akc Gazette, 125(2): 28-29, 2008.

Gross, T.L.; Ihrke, P.J.; Walder, E.J. Veterinary dermatopathology: a macroscopic and microscopic evaluation of canine and feline skin diasease. Saint Louis: Mosby Year Book, 2009. 256p.

Gross, T.L.; Ihrke, P.J.; Walder, E.J. Skin diseases of the dog and cat. Clinical and histopatological diagnosis. Oxford: Blackwell Science, 2005. 563p.
Lam, A.T.; Affolter, V.K.; Outerbridge, C.A.; Gericota, B.; White, S.D. Oral vitamin A as an adjunct treatment for canine sebaceous adenitis. Veterinary Dermatology, 22: 301311, 2011 .

Larsson, C.E.; Lucas, R. Tratado de medicina externa: dermatologia veterinária. São Caetano do Sul: Interbook, 2016. 888 p.

Linek, M. Adenite sebácea em cães. Veterinary Focus, 18: 12-16, 2008.

Lucas, R.; Castangalo, K.L.; Rolan, R.T.; Jericó, M.M. Adenite sebácea: nova opção terapêutica - relato de caso. Revista Nosso Clínico, 11: 8-10, 2008.

Lowell, A. What's new in veterinary dermatology. In: WORLD SMALL ANIMAL VETERINARY ASSOCIATION, 30th. 2005. Mexico. Proceedings... Mexico, 2005.

Medleau, L.; Hnilica, K.A. Dermatologia de pequenos animais. São Paulo: Roca, 2009. $512 \mathrm{p}$.

Miller, W.H.J.; Griffin, C.E.; Campbel, K.L. Miscellaneous skin diaseases. Small Animal Dermatology. Missouri: Elsevier, 2013, 948p.

Obladen, A.; Farias, M.; Choque, K.C.C.; Werner, J.; Tammenhain, B. Adenite sebácea em um cão da raça lhasa apso. Acta Scientiae Veterinariae, 35: 448-449, 2007.

Reichler, I.M.; Hauser, B.; Schiller, I. Sebaceous adenitis in the Akita: clinical observations, histopathology and heredity. Veterinary Dermatology, 12: 243-253, 2001.

Reis, F.M. Adenite sebácea granulomatosa em um cão sem raça definida: relato de caso. 2009. 20 f. Monografia (Especialização). Clínica Médica de Pequenos Animais, Universidade Federal Rural do Semi-Árido, Mossoró, 2009.

Rhodes, K.H.; Codner, E.C. Dermatologia de pequenos animais - Consulta em cinco minutos. Rio de Janeiro: Revinter, 2005. $553 \mathrm{p}$.

Scott, D.W., Miller, W.H.; Griffin, C.E. Miscellaneous skin diseases. In: Patel, A.; Forsythe, P. Small animal dermatology. Saunders: Elsevier, 2001, 379p.

Scott, D.W.; Miller, W.H.; Grifin, C.E. Dermatologia de pequenos animais. Rio de Janeiro: Interlivros, 1996. 856p. 
Scott, D.W. Sterile granulomatous sebaceous adenitis in dogs and cats. Veterinary Anne, 33: 236, 1993.

Sousa, C.A. Sebaseous adenitis. Veterinary Clinical. Small Animal, 36: 243-249, 2006.

Travell, E., Bergvall, K., Egenvall, A. Sebaceous adenitis in Swedish dogs, a retropective study of 104 cases. Acta Veterinaria Scandinavica, 50: 11, 2008.

Vercelli, A.; Cornegliani, L; Tronca, L. Sebaceous adenitis in three related Hovawart dogs. Veterinary Dermatology, 15: 52, 2004.
White, S.D.; Linder, K.E.; Schultheiss, P. Sebaceous adenitis in four domestic rabbits (Oryctatagus cuniculus). Veterinary Dermatology, 11: 53-60, 2000.

White, S.D.; Rosychuk, R.; Scott, K.V.; Hargis, A.M.; Jonas, L.; Trettien, A. Sebaceous adenitis in dogs and results of treatment with isotretinoin and etretinate: 30 cases (19901994). Journal of the American Veterinary Medical Association, 207: 197200, 1995. 